\title{
Functional Graphene oxide and role of hydroxyl groups on humidity sensing
}

\author{
Qawareer Fatima ${ }^{1,2, *}$, Azhar Ali Haidry ${ }^{1,2, *}$, Linchao Sun ${ }^{1,2}$, Zhong Li $^{1,2}$, Lijuan Xie ${ }^{1,2}$, Zhengjun Yao ${ }^{1,2}$ \\ ${ }^{1}$ College of Materials Science and Technology, Nanjing University of Aeronautics and Astronautics, \\ 211100 Nanjing, China \\ ${ }^{2}$ Key Laboratory of Materials Preparation and Protection for Harsh Environment, Ministry of Industry \\ and Information Technology, 211100 Nanjing, China \\ *Corresponding author's e-mail address: Qawareer.fatima@yahoo.com, aa.haidry@nuaa.edu.cn,
}

\begin{abstract}
IMCS2018_Headline"):
In this work we prepared Graphene oxide with modified Hummers method. We study the effect of hydroxyl groups on the humidity sensing properties at room temperature with low voltage of $0.1 \mathrm{~V}$ and fast response time. The functionality of the GO was intensively studied by using Fourier-transform infrared spectroscopy. We performed intensive gas sensing and electrical measurements coupled with mandatory XRD, SEM, FTIR and Raman experiment which show promising results. The analysis shows that the enhanced humidity performance is given by the presence of surface hydroxyls groups due to functionalize Graphene oxide.
\end{abstract}

Key words: Graphene oxide, humidity sensor, power consumption, hydroxyl groups, sensing mechanism.

\section{Introduction}

Gas sensor is a device that detects the presence or leakage of gases in a system. Since the last two decades, the demand of gas sensors has been increasing in industrial applications such as environmental monitoring, health issues, public and home safety, combustion engines, and in process control. Major scientific communities around the globe are putting great efforts to develop new smart gas sensors to meet this demand.

Graphene Oxide (GO), reduced Graphene oxide (rGO) and functionalized Graphene oxide (fGO) are derivative of Graphene and have inadmissible defects which surprisingly give rise to exciting properties [1,2]. Notwithstanding Graphene is durable material but can be fragile under some environments, on the other hand GO exhibits plastic deformation before breaking. GO also dissolves easily in water and other organic solvent due to its internal oxygen functional groups [3].

The sensors used to monitor humidity play increasingly important role not only in the industry but to our environment also. Humidity is present in almost every industrial process and sometimes can cause poisoning to the surface of applied materials. Moreover, humidity sensors are used in many applications in the field of applied materials processing, food processing and preservation, healthcare, smart home and industrial fabrication. In a very recent report, our reported the increased performance of humidity sensors based on fiber optic coupled with GO film [4]. The sensors can monitor the wide range of humidity, however; failed to show response below $30 \% \mathrm{RH}$.

In the current report we study the effect of hydroxyl groups on the room temperature humidity sensing characteristics of GO fabricated with modified Hummers method. The sensors show promising sensing ability to be operated even at $0.1 \mathrm{~V}$ applied voltage. This way the power consumption of the sensor can be reduced significantly.

\section{Experimental}

Firstly, GO was prepared by modified Hummers method. During low-temperature stage, potassium permanganate $(1.5 \mathrm{~g})$ was added to a vigorously stirring, ice-cooled mixture of graphite $(1 \mathrm{~g})$, sulfuric acid $(90 \mathrm{~mL})$ and sodium nitrate $(1 \mathrm{~g})$. After $2 \mathrm{~h}$ continuous stirring the solution was removed from ice bath and stirred at $35{ }^{\circ} \mathrm{C}$ for $1 \mathrm{~h}$. Followed by the addition of hydrogen peroxide $(30 \% / 20 \mathrm{~mL})$ and $\mathrm{DI}$ water 
(200 mL) under continue stirring for $1 \mathrm{~h}$ at 90 ${ }^{\circ} \mathrm{C}$. Subsequently, the solution was washed with $5 \% \mathrm{HCl}$ solution and $\mathrm{DI}$ water followed by overnight drying procedure at room temperature. Subsequently, GO $(10 \mathrm{mg})$ was dispersed in ethanol $(20 \mathrm{~mL})$. Further, the effect of ether contents on various properties was investigated. The dispersion was drop-casted onto the substrate having already gold metal electrodes with spacing of about 300 micrometers.

Addition FTIR, XRD, AFM and gas sensing tests were performed to analyze the surface functionality, crystal structure, surface topography and humidity sensing ability. The details of theses analysis can be found elsewhere in another report [4].

\section{Results}

The overall sensing characteristics of sensors prepared in this work show excellent sensing characteristics. The best humidity performance at room temperature was obtained with sensors prepared with $5 \mathrm{ml}$ ether as demonstrated in Fig. 1. To understand the role of hydroxyl groups, the intensive FTIR analysis were performed, which shows that the amount of $\mathrm{OH}^{-1}$ groups increases with increasing ether contents, see Fig. 2. Thus this results in enhancement of humidity sensing at room temperature.

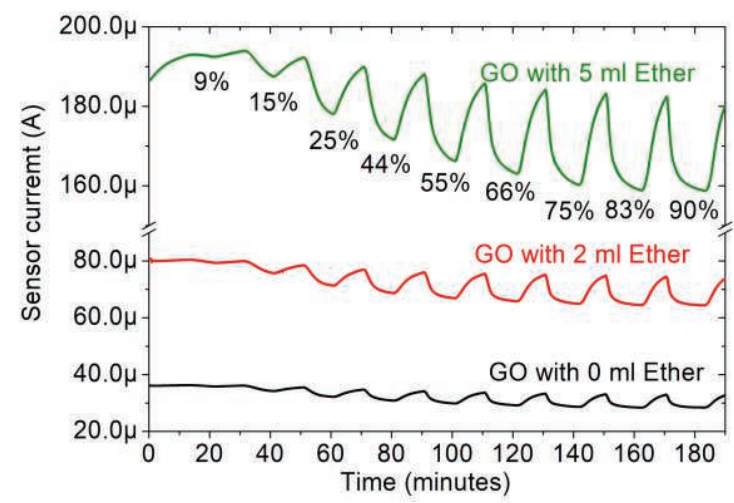

Fig. 1. Typical dynamic humidity responses of sensors with various ether contents measured with $0.1 \mathrm{~V}$ at room temperature.

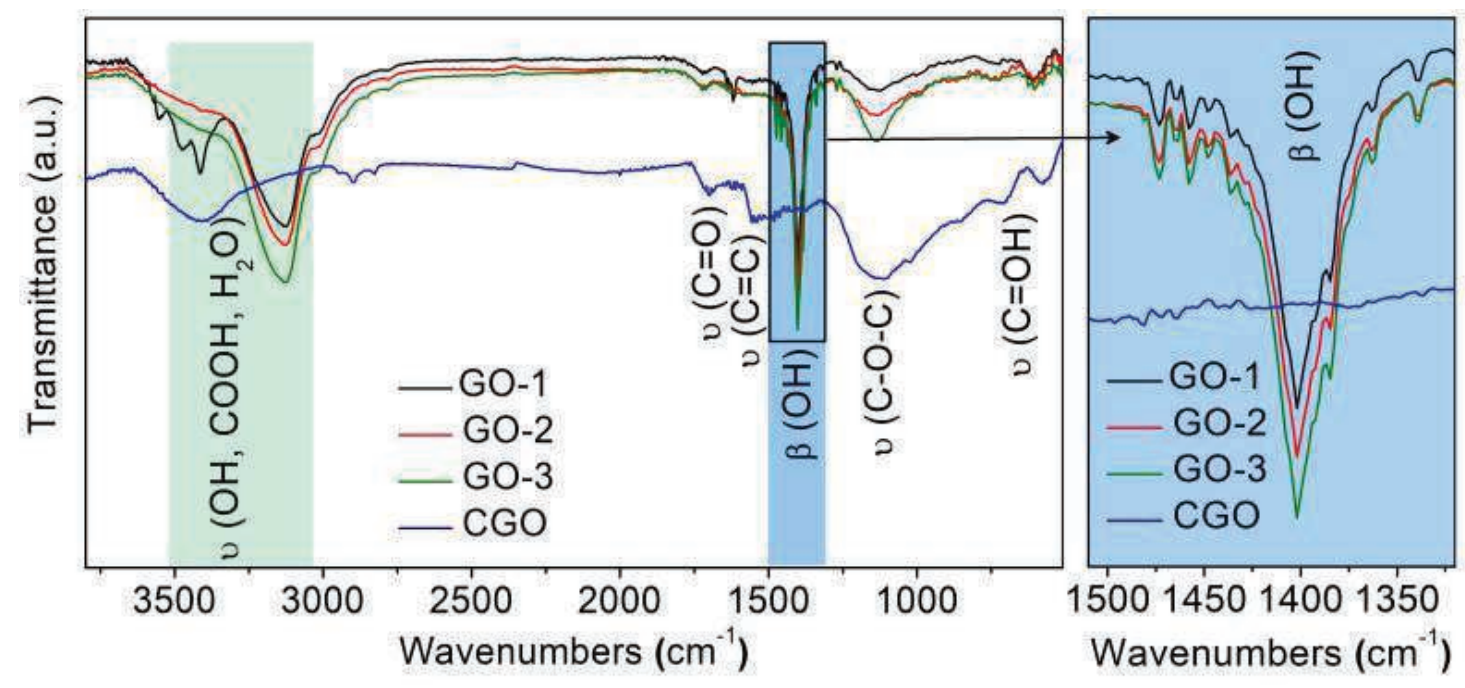

Fig.2. The FTIR spectra of CGO, GO with $3 \mathrm{ml}$ (GO1), $6 \mathrm{ml}$ (GO2) and $12 \mathrm{ml}$ (GO3) KMnO4.

\section{References}

[1] C. Y. Lee, G. B. Lee, Humidity Sensors: A Review, Sensor Lett., 3 (2005) 1-14. doi:10.1166/sl.2005.045

[2] C. Laville, C. Pellet, Interdigitated humidity sensors for a portable clinical microsystem, IEEE transactions on bio-medical engineering, 49 (2002) 1162-1167. DOI: 10.1109/TBME.2002.802052

[3] G. P. Alcantara, L. E. B. Ribeiro, A. F. Alves, C. M. G. Andrade, F. Fruett, Humidity sensor based on zeolite for application under environmental conditions, Micropor. Mesopor. Mater., 247
(2017) 38-45.

https://doi.org/10.1016/j.micromeso.2017.03.042

[4] Y.R. Choi et al., Role of oxygen functional groups in graphene oxide for reversible room temperature NO2 sensing, Carbon vol. 91 (2015), pp. $178-187$. https://doi.org/10.1016/j.carbon.2015.04.082

[5] Linchao Sun et al., Improving the humidity sensing below $30 \% \mathrm{RH}$ of $\mathrm{TiO} 2$ with $\mathrm{GO}$ modification, Materials Research Bulletin 99 (2018) 124-131. https://doi.org/10.1016/j.materresbull.2017.11.00 1 